\title{
LA EFECTIVIDAD DEL DERECHO DE LA UNIÓN EUROPEA COMO MOTIVO DE PROTECCIÓN DE LA COSA JUZGADA NACIONAL: NOTA SOBRE LA SENTENCIA DE 24 DE OCTUBRE DE 2018, XC Y OTROS
}

\author{
ARACELI TURMO' \\ Universidad de Nantes (Francia) \\ araceli.turmo@univ-nantes.fr
}

Cómo citar/Citation

Turmo, A. (2019).

La efectividad del derecho de la Unión Europea como motivo de protección de la cosa juzgada nacional: nota sobre la sentencia de 24 de octubre de 2018, XC y otros. Revista de Derecho Comunitario Europeo, 63, 599-626 https://doi.org/10.18042/cepc/rdce.63.07

\section{Resumen}

La sentencia del Tribunal de Justicia de 24 de octubre 2018, XC y otros, presenta una reflexión interesante sobre la utilidad de extender al ámbito del derecho de la Unión los mecanismos que, en algunos Estados miembros, permiten repetir procedimientos penales en caso de violación del Convenio Europeo de Derechos Humanos $(\mathrm{CEDH})$. El Tribunal de Justicia presenta un razonamiento persuasivo que se apoya, por una parte, en la necesidad de respetar la cosa juzgada como principio de derecho interno y, por otra parte, en la suficiente efectividad del sistema jurisdiccional de la Unión Europea, en oposición al del CEDH.

1 Profesora de Derecho Público, Derecho de la Unión Europea, en la Facultad de Derecho de la Universidad de Nantes (Francia). 


\section{Palabras clave}

Convenio Europeo de Derechos Humanos; cosa juzgada; derecho procesal; principio de efectividad; principio de equivalencia; Tribunal de Justicia.

\section{THE EFFECTIVENESS OF EUROPEAN UNION LAW AS A REASON TO PROTECT NATIONAL RES JUDICATA: NOTE ON THE JUDGMENT OF 24 OCTOBER 2018, XC AND OTHERS}

\section{Abstract}

The ruling made by the Court of Justice on 24 October 2018, in case $X C$ and others, presents an interesting reflection on the usefulness of extending the scope of the mechanisms which, in certain Member States, allow for criminal proceedings to be re-opened in case of a violation of the ECHR, to European Union Law. The Court of Justice presents a convincing reasoning based on, on the one hand, the need to respect res judicata as a principle of national law and, on the other hand, on the sufficient effectiveness of the European Union's judicial system, as opposed to that of the ECHR.

\section{Keywords}

Court of Justice; European Convention on Human Rights; principle of effectiveness; principle of equivalence; procedural law; res judicata.

\section{L'EFFECTIVITÉ DU DROIT DE L'UNION EUROPÉENNE COMME MOTIF DE PROTECTION DE LA CHOSE JUGÉE NATIONALE: NOTE SUR L'ARRÊT DU 24 OCTOBRE 2018, XC ET AUTRES}

\section{Résumé}

L'arrêt rendu par la Cour de justice le 24 octobre 2018, dans l'affaire XC et autres, présente une réflexion intéressante sur l'utilité d'étendre le champ d'application des mécanismes qui, dans certains États membres, permettent de rouvrir des procédures pénales en cas de violation de la $\mathrm{CEDH}$, au droit de l'Union européenne. La Cour de justice présente un raisonnement convaincant fondé, d'une part, sur la nécessité de respecter l'autorité de la chose jugée comme principe de droit interne et, d'autre part, sur l'effectivité suffisante du système juridictionnel de l'Union européenne, par opposition à celui de la $\mathrm{CEDH}$.

\section{Mots clefs}

Autorité de la chose jugée; Convention Européenne des Droits de l'Homme; Cour de Justice; droit processuel; principe d'effectivité; principe d'équivalence. 


\section{SUMARIO}

I. INTRODUCCIÓN. II. UN BALANCE LEGÍTIMO Y PREDECIBLE ENTRE COSA JUZGADA Y EFECTIVIDAD DEL DERECHO DE LA UE: 1. La importancia de la cosa juzgada nacional en el derecho de la UE. 2. Las limitaciones suficientes a la cosa juzgada nacional en el derecho de la UE. III. UNA EXPOSICIÓN DE LAS DIFERENCIAS ESENCIALES ENTRE LA EFECTIVIDAD DE LOS SISTEMAS JURISDICCIONALES DE LA UE Y DEL CEDH: 1. La justificación del procedimiento de revisión por las características específicas del sistema jurisdiccional del CEDH. 2. Las diferencias fundamentales entre el sistema jurisdiccional de la UE y el del CEDH. IV. CONCLUSIÓN. BibLIOGRAFía.

\section{INTRODUCCIÓN}

En la jurisprudencia del Tribunal de Justicia de la Unión Europea, la efectividad del derecho de la Unión Europea (UE) ha frecuentemente sido presentada como un motivo de limitación de los efectos de la cosa juzgada nacional. Una sentencia de octubre de 2018 demuestra que los dos principios no son tan opuestos como ha podido parecer y se basa en el nivel de efectividad que garantiza el sistema jurisdiccional de la Unión para rechazar una opción que hubiera antepuesto la aplicación correcta del derecho de la Unión a la firmeza de las resoluciones judiciales nacionales.

La sentencia de 24 de octubre de 2018, XC y otros ${ }^{2}$ dio por primera vez al Tribunal de Justicia la oportunidad de analizar un mecanismo procesal nacional que permite repetir un procedimiento penal en caso de violación del Convenio Europeo de Derechos Humanos (CEDH) desde el punto de vista del derecho de la UE. Tal como demuestra un estudio encargado por el Comité de Expertos para la reforma del Tribunal (DH-GDR, 2016), tales

2 Sentencia del Tribunal de Justicia de 24 de octubre de 2018, XC y otros, C-234/17, EU:C:2018:853. 
mecanismos existen en España ${ }^{3}$ y en varios otros Estados miembros ${ }^{4}$ para garantizar la efectividad de las sentencias del Tribunal Europeo de Derechos Humanos (TEDH) y, sobre todo, la protección de los derechos de las víctimas de violaciones identificadas por este Tribunal. Estos mecanismos están justificados por la necesidad de remediar violaciones graves que, debido a la naturaleza del sistema jurisdiccional del $\mathrm{CEDH}$, se convertirían en definitivas si no fuera posible repetir el procedimiento penal. La importancia de la protección de los derechos fundamentales, especialmente en materia penal, puede en estos casos justificar una excepción al carácter definitivo de una sentencia judicial firme.

La cosa juzgada no está prevista por las fuentes escritas del derecho de la Unión, pero fue reconocida como un componente del derecho procesal de la Unión desde los primeros años de la construcción comunitaria ${ }^{5}$, más recientemente, como un principio, basado principalmente en el de seguridad jurídica ${ }^{6}$, cuya importancia es equivalente en los ordenamientos jurídicos de la Unión y de los Estados miembros 7 . Sin embargo, el Tribunal de Justicia ha

3 España introdujo en 2015 un recurso de revisión que permite la reapertura de procedimientos internos tras una sentencia condenatoria del TEDH: art. 5 bis de la Ley Orgánica del Poder Judicial introducido por el apdo. tres del art. único de la L.O. 7/2015, de 21 de julio, por la que se modifica la L.O. 6/1985, de 1 de julio, del Poder Judicial (BOE, 22 de julio de 2015) y art. 954.3 de la Ley de Enjuiciamiento Criminal, redactado por el apdo. quince del art. único de la Ley 41/2015, de 5 de octubre, de modificación de la Ley de Enjuiciamiento Criminal para la agilización de la justicia penal y el fortalecimiento de las garantías procesales (BOE, 6 de octubre de 2015).

4 Por ejemplo, Dinamarca, Estonia, Finlandia, Francia, Italia.

5 Debido a la ausencia de fuentes escritas combinada con la evidencia con la cual se impuso la necesidad de la cosa juzgada en el derecho procesal comunitario, las primeras sentencias del Tribunal de Justicia que aplican el principio lo hacen de forma casi implícita: sentencias del 13 de julio de 1961, Raymond Elz, 22/60 y 23/60, EU:C:1961:17; y del 16 de diciembre de 1963, Acciaierie San Michele, 2-10/63, EU:C:1963:59.

6 La clasificación de la cosa juzgada como principio aparece por primera vez en el derecho de la Unión en la Sentencia del Tribunal de Justicia de 1 de junio de 1999, Eco Swiss, C-126/97, EU:C:1999:269.

7 El régimen de la cosa juzgada en el derecho de la UE es distinto al que existe en España. La influencia principal en las primeras etapas de la construcción de las normas relevantes en el derecho procesal de la Unión fue la del derecho francés, por ejemplo con la distinción fuerza de cosa juzgada / autoridad de la cosa juzgada, y los criterios de la «triple identidad» de partes, objeto y causa del procedimiento que son reproducidos de forma idéntica a la del derecho procesal francés (por ejemplo, Sentencia del Tribunal de Primera Instancia del 5 de junio de 1996, NMB c/ Comisión, T-162/94, 
indicado en algunas ocasiones que la exigencia de efectividad del derecho de la UE podía así mismo conllevar limitaciones o excepciones a la aplicación de las normas que garantizan el respeto de la cosa juzgada en los Estados miembros.

Por este motivo, la cuestión de la pertinencia de mecanismos similares que permitan repetir procedimientos judiciales en casos de violaciones del derecho de la Unión parece pertinente. Si tales mecanismos ya existen en un Estado miembro, ¿por qué no usarlos para remediar una violación grave del derecho de la Unión? Esta pregunta es exactamente la que hizo al Tribunal de Justicia el Oberster Gerichsthof (Tribunal Supremo de lo Civil y Penal) austríaco en el asunto $X C$ y otros. El litigio principal se centra en las solicitudes de asistencia judicial en materia penal presentadas a las autoridades austriacas por la fiscalía del cantón de Saint Gall, en Suiza, durante una investigación sobre un presunto fraude fiscal. Los tres interesados interpusieron en Austria varios recursos para impugnar la realización de sus audiciones por la fiscalía local, alegando que procedimientos penales concluidos en Alemania y en Liechtenstein durante los dos años anteriores impedían que fueran procesados de nuevo por tales sospechas, en razón del art. 54 del Convenio de aplicación de Schengen (CAAS) que consagra el principio non bis in idem. El Oberlandesgericht Innsbruck (Tribunal Superior regional de Innsbruck) consideró en última instancia que no existían motivos para identificar una violación del art. 54 del CAAS. Esta resolución, dictada en última instancia, es firme y se beneficia de los efectos de la cosa juzgada.

Sin embargo, los interesados solicitaron al Oberster Gerichsthof la repetición del procedimiento penal al amparo del art. 363a de la Ley de Enjuiciamiento Penal. Alegaban que la desestimación de las solicitudes de asistencia judicial vulneraba derechos reconocidos no solo por el CEDH sino también por el CAAS y por la Carta de los Derechos Fundamentales de la Unión Europea. El art. 363a permite la revisión de una sentencia y la repetición de un procedimiento penal cuando, mediante una sentencia del TEDH, queda claro que la sentencia contiene una violación del CEDH y que no se puede excluir que esa violación haya podido influir en el contenido de la resolución en perjuicio del afectado. En principio, pues, esta disposición solo se puede

EU:T:1996:71, apdo. 37). Otras influencias aparecen, por ejemplo la española, en las conclusiones del abogado general Ruiz-Jarabo Colomer con la expresión «efectos de la cosa juzgada material y formal» (Conclusiones presentadas el 16 de diciembre de 2004, en el asunto Bélgica c/ Comisión, C-110/03, EU:C:2004:815, apdo. 30). Los tribunales de la Unión Europea han construido un régimen mayoritariamente influenciado por el derecho procesal francés, pero que no es idéntico y es ante todo adecuado para el derecho procesal de la Unión: para un análisis exhaustivo, véase Turmo (2017). 
emplear en caso de violación de derechos garantizados por el CEDH reconocida por el TEDH o, según la jurisprudencia del Oberster Gerichsthof, incluso antes si el propio tribunal reconoce la violación. La utilización del art. 363a, o de una disposición similar, para poner remedio a una violación del derecho de la Unión no está prevista en la legislación austriaca. El Oberster Gerichsthof quiso asegurarse de que esta situación era compatible con las exigencias del derecho de la Unión, en particular con los principios de cooperación leal, de equivalencia y de efectividad.

El Gobierno austriaco propuso una excepción de inadmisibilidad, basada en los argumentos de que el art. 363a no forma parte del ámbito de aplicación del derecho de la Unión y de que la cuestión no era pertinente puesto que la jurisprudencia nacional ya había considerado que el art. 54 del CAAS constituía una base jurídica suficiente para solicitar la repetición del procedimiento penal al amparo de dicha disposición ${ }^{8}$. El Tribunal descartó la excepción rápidamente: consideró que, al margen de la naturaleza de la vía de recurso contemplada, la situación que ha dado origen al litigio principal está comprendida en el ámbito de aplicación del derecho de la Unión? ${ }^{2}$ Además, reiterando su jurisprudencia consolidada sobre la admisibilidad de las cuestiones relativas a la interpretación del derecho de la Unión, recordó que estas disfrutan de una presunción de pertinencia ${ }^{10} \mathrm{y}$ aceptó las razones expuestas por el tribunal remitente para justificar la necesidad de la cuestión.

La respuesta del Tribunal de Justicia sobre el fondo, muy similar a la que había propuesto su abogado general ${ }^{11}$, es una afirmación muy clara de que no existe ninguna obligación de ampliar el ámbito de aplicación de este tipo de recurso para aplicarla a las violaciones del derecho de la Unión. La sentencia del Tribunal de Justicia está basada en una aplicación convencional de los principios de equivalencia y de efectividad, expresiones del principio de cooperación leal en materia procesal cuando no ha habido armonización de una regla específica. Tal como recuerda el Tribunal de Justicia ${ }^{12}$, las modalidades de aplicación del principio de la cosa juzgada se rigen por el ordenamiento jurídico de cada Estado miembro al no existir una normativa

Apdos. 13 y 15 de la sentencia, respectivamente.

Apdo. 14.

10 Apdos. 16-18. El Tribunal de Justicia cita una sentencia reciente (27 de junio de 2018, Altiner y Ravn, C-230/17, EU:C:2018:497), pero la presunción de pertinencia de las decisión de remisión está muy bien establecida: Sentencia del Tribunal de Justicia del 7 de septiembre de 1999, Beck y Bergdorf, C-355/97, EU:C:1999:391, apdo. 22.

11 Conclusiones del abogado general Saugmandsgaard Øe, XC y otros, C-234/17, EU:C:2018:391.

12 Apdo. 21 de la sentencia. 
de la Unión, en virtud del principio de autonomía procesal, aunque autores como Bobek (2012: 316 y ss.) pongan legítimamente en duda la existencia y el ámbito de esta noción como principio de derecho de la Unión. Ahora bien, en este caso el art. 363a constituye una excepción a la cosa juzgada, justificada en el ordenamiento jurídico austriaco por motivos particulares: la efectividad de la protección de los derechos fundamentales por el TEDH necesitaría este tipo de mecanismo. La cuestión prejudicial equivale a preguntarse si la efectividad de la protección de los derechos fundamentales garantizada por el derecho de la Unión también necesita tales excepciones a la cosa juzgada.

El Oberster Gerichsthof interroga al Tribunal de Justicia sobre la compatibilidad del ámbito de aplicación de una disposición tal como el art. 363a con el art. 4 TUE, apdo. 3, «en relación con los principios de equivalencia y de efectividad que del mismo se deducen ${ }^{13}$. La asociación de estos dos principios con el de cooperación leal que establece el art. 4 TUE no es necesaria, ya que puede considerarse su expresión en el ámbito del derecho procesal ${ }^{14}$. La justificación de estos límites a la autonomía procesal de los Estados miembros es precisamente la cooperación leal que se impone a los sistemas judiciales a fin de asegurar la efectividad del derecho de la Unión ${ }^{15}$. De ahí que la cuestión sea reformulada inmediatamente por el Tribunal como una pregunta que trata directamente de las exigencias derivadas de los principios de equivalencia y de efectividad ${ }^{16}$. El Tribunal ofrece, pues, una evaluación de la compatibilidad del recurso nacional con cada uno de estos principios sucesivamente, citando jurisprudencia antigua y recordando sentencias más recientes que ofrecen más detalles sobre el tipo de análisis que debe llevar a cabo, en particular, la insistencia con que se debe tomar en cuenta el lugar que ocupa la norma procesal en el conjunto del procedimiento y el desarrollo de este ${ }^{17}$. El razonamiento desarrollado en relación con cada principio se apoya a su vez en características constitucionales del derecho de la Unión y en la concepción del sistema

\footnotetext{
Apdo. 11.

Apdo. 22.

15 La asociación de la cooperación leal con los principios de efectividad y de equivalencia ya había aparecido en la jurisprudencia (por ejemplo, en la Sentencia de 4 de octubre de 2012, Byankov, C-249/11, EU:C:2012:608, apdos. 69 y ss.), pero no es sistemática. La cooperación leal ha sido empleada alternativamente con el principio de primacía (o efectividad) del derecho de la Unión para justificar intervenciones en los derechos procesales de los Estados miembros. Esta indecisión en la jurisprudencia no parece, sin embargo, tener consecuencias y la motivación del Tribunal de Justicia parece siempre la misma.

6 Apdo. 20 de la sentencia.

17 Apdos. 22-24.
} 
judicial de la Unión que el Tribunal defiende con determinación creciente desde hace algunos años ${ }^{18}$.

El Tribunal de Justicia asienta su motivación en dos líneas argumentativas. En primer lugar, confirma que el derecho de la Unión respeta la cosa juzgada como principio de derecho nacional (I). Este respeto debe siempre conducir a limitar las exigencias derivadas del principio de efectividad del derecho de la UE en relación con la corrección de violaciones causadas por resoluciones judiciales firmes. En segundo lugar, el Tribunal de Justicia se basa en las diferencias esenciales entre los sistemas judiciales asociados con la UE y con el CEDH para establecer que tales mecanismos nacionales no causan violaciones del principio de equivalencia, o más bien, que la prueba de equivalencia no es pertinente en tales casos (II).

\section{UN BALANCE LEGÍTIMO Y PREDECIBLE ENTRE COSA JUZGADA Y EFECTIVIDAD DEL DERECHO DE LA UE}

El principio de efectividad plantea los interrogantes más próximos a los objetivos concretos del art. 363a y de mecanismos similares en otros Estados europeos. La finalidad de estos mecanismos es garantizar una mayor efectividad del CEDH tal como lo interpreta el TEDH, permitiendo revisar sentencias judiciales firmes que se basan en violaciones de derechos consagrados en el Convenio. Así pues, tienen dos objetivos: evitar que violaciones contenidas en sentencias judiciales se puedan mantener o incluso extender en los ordenamientos jurídicos nacionales, pero ante todo asegurar la protección de las víctimas de tales violaciones (Imbert, 2007: 59) ${ }^{19}$. Estos objetivos pueden sin duda existir en el ordenamiento jurídico de la UE. La búsqueda de la efectividad máxima para las normas de derecho de la Unión y la jurisprudencia desarrollada por el Tribunal de Justicia podrían justificar soluciones similares. Sin embargo, la efectividad del derecho de la Unión no es un objetivo absoluto, y debe ponderarse siempre en relación con otros objetivos que pueden justificar, desde el punto de vista de los Estados miembros, ciertos obstáculos para esta efectividad. En este caso, el obstáculo más evidente es el principio de la cosa juzgada. El Tribunal de Justicia ha reconocido su importancia tanto en los ordenamientos jurídicos nacionales como en el de la Unión y ha admitido,

18 La importancia del sistema jurisdiccional de la UE en la motivación en el razonamiento del Tribunal aparece claramente en los dictámenes 1/09 (de 8 de marzo de 2011, EU:C:2011:123) y 2/13 (de 18 de diciembre 2014, EU:C:2014:2454), por ejemplo.

19 Apdo. 59 de la sentencia. 
salvo en circunstancias excepcionales, que pueda constituir un obstáculo para la plena aplicación del derecho de la Unión. Esta sentencia confirma el respeto expresado por el Tribunal de Justicia acerca de la cosa juzgada como principio de derecho de la Unión (1). El Tribunal va más allá y demuestra por qué la efectividad del derecho de la Unión no requiere de tales limitaciones a la cosa juzgada nacional (2).

\section{LA IMPORTANCIA DE LA COSA JUZGADA NACIONAL EN EL DERECHO DE LA UE}

La firmeza de las afirmaciones del Tribunal de Justicia reiterando su respeto hacia la cosa juzgada nacional puede sorprender a lectores familiarizados con una parte de su jurisprudencia relativa a la cosa juzgada. En efecto, algunas sentencias, sobre todo a partir de Köbler ${ }^{20}$ y Lucchini ${ }^{21}$, han dado lugar a reacciones que denotan inquietud. La «saga» de la jurisprudencia en torno a la cosa juzgada italiana es el ejemplo más destacado de lo que algunos habían considerado incursiones indebidas en el derecho procesal de los Estados miembros (Bříza, 2008; Wattel, 2004). Sin embargo, la sentencia $X C$ y otros demuestra que el temor a que la injerencia del Tribunal de Justicia conduzca a restricciones excesivas de la cosa juzgada no estaba justificado.

La jurisprudencia del Tribunal de Justicia sobre la cosa juzgada nacional, como en lo que se refiere a otros aspectos del derecho procesal de los Estados miembros, sigue estando guiada por dos consideraciones generales. Por una parte, desde el punto de vista de la Unión, el sistema judicial de cada Estado miembro es un instrumento que debe ponerse al servicio de la aplicación efectiva del derecho de la Unión y de la protección de los derechos que este sistema reconoce a los justiciables. Por otra parte, los Estados miembros conservan, en principio, la libertad de elegir el sistema judicial y el derecho procesal que les convenga, y la buena voluntad de los tribunales nacionales es esencial para asegurar su cooperación con el Tribunal de Justicia y, por tanto, la aplicación del derecho de la Unión. Estas consideraciones aparecen en la jurisprudencia bajo distintas formas, principalmente como una oposición entre el principio de efectividad del derecho de la Unión y el de autonomía procesal de los Estados miembros (Bobek, 2012: 315-320). El resultado es, por lo general, la ausencia de intervención en el derecho procesal nacional. Sin embargo, cuando el Tribunal considera que la aplicación efectiva del derecho de la Unión no es posible debido a una norma de derecho procesal, no duda en

20 Sentencia de 30 de septiembre de 2003, Köbler, C-224/01, EU:C:2003:513.

21 Sentencia de 18 de julio de 2007, Lucchini SpA, C-119/05, EU:C:2007:434. 
exigir una modificación o la inaplicación de aquella norma (Adinolfi, 2012: 287). Los principios de equivalencia y de efectividad, cuyo contenido es determinado por el mismo Tribunal de Justicia, le permiten exigir tales medidas en aquellos casos.

La jurisprudencia sobre la cosa juzgada nacional ofrece una buena ilustración de este tipo de razonamiento. Como recuerda el Tribunal, por lo general nunca exige que el juez nacional deje inaplicada una regla que protege la cosa juzgada, aunque esto limite la efectividad del derecho de la Unión - salvo en los casos en los que el Tribunal decide que esta restricción a la efectividad es excesiva-. Así pues, desde el punto de vista del Tribunal de Justicia, sus sentencias sobre la cosa juzgada italiana solo exigen una adaptación del derecho procesal nacional en un sentido que permite un nivel de efectividad razonable para el derecho de la Unión.

La jurisprudencia del Tribunal de Justicia también indica que hay que aprovechar todas las opciones que existen en derecho interno: cuando el derecho procesal del Estado permite limitar el impacto de la cosa juzgada sobre la efectividad del derecho de la Unión, esta opción se torna en obligación ${ }^{22}$. Esta posición se justifica por una lógica similar: dado que la aplicación efectiva del derecho de la Unión siempre es la prioridad desde el punto de vista del Tribunal de Justicia, hay que aprovechar todas las opciones que existen dentro del marco del derecho procesal nacional para lograrla. Sin embargo, dado que la cosa juzgada es un componente esencial de todo sistema jurisdiccional, debe ser respetada y, por lo general, el derecho de la Unión no debe imponer excepciones a la aplicación de las normas nacionales que aseguran su respeto.

En efecto, frente a la efectividad del derecho de la UE, la jurisprudencia del Tribunal de Justicia sobre la cosa juzgada añade el principio de seguridad jurídica al clásico de la autonomía procesal. Este tipo de razonamiento permite al Tribunal de Justicia insistir en que el derecho de la Unión respeta los sistemas judiciales nacionales y a los tribunales nacionales, mediante el acercamiento del derecho procesal de la UE a los de los Estados miembros en lo que se refiere a la importancia de los principios de la cosa juzgada y de su fundamento, la seguridad jurídica ${ }^{23}$. Así pues, el Tribunal afirma con frecuencia que "procede

22 Sentencia del Tribunal de Justicia de 13 de enero de 2004, Kühne and Heitz, C-453/00, EU:C:2004:17, apartados 24-26.

23 Sentencia Eco Swiss (cit. supra, nota 6), apdo. 46. Dado que la construcción de la cosa juzgada en el derecho de la Unión tuvo lugar bajo la influencia mayoritaria del derecho francés, la tutela judicial efectiva nunca ha sido reconocida como fundamento legal de la cosa juzgada como lo es en el derecho español (STC 43/85, de 22 de marzo de 1985, BOE n. ${ }^{\circ}$ 94, 19/4/1985); véase Rubio Garrido (2002: 264). 
recordar la importancia que tiene, tanto en el ordenamiento jurídico de la Unión como en los ordenamientos jurídicos nacionales, el principio de la cosa juzgada ${ }^{24}$, afirmación a veces asociada con referencias directas o indirectas a la seguridad jurídica o a la recta administración de la justicia ${ }^{25}$. Puesto que las mismas bases justifican la existencia y la importancia de la cosa juzgada en todo sistema jurisdiccional, el Tribunal de Justicia asimila el principio que ha introducido él mismo en el derecho procesal de la Unión al que existe en los ordenamientos jurídicos nacionales como prueba de su buena voluntad hacia los derechos procesales de los Estados miembros ${ }^{26}$. Cabe notar que las primeras incidencias de esta aproximación de los derechos procesales nacionales y europeo en lo que se refiere a la cosa juzgada aparecieron justamente en sentencias que imponían límites a la aplicación normal de la cosa juzgada nacional con motivo de la aplicación correcta del Derecho de la Unión ${ }^{27}$. Es posible dudar de la sinceridad de la aproximación puesto que el derecho procesal de los Estados miembros siempre queda sometido a las exigencias de efectividad y equivalencia y, por ello, a las exigencias impuestas por el derecho de la Unión. Sin embargo, esta frase se encuentra ahora también en sentencias relativas a la cosa juzgada en el contexto del derecho procesal de la Unión ${ }^{28}$ y se puede considerar la expresión de una voluntad real de respetar lo que es un principio fundamental de todo derecho procesal europeo.

La misma técnica de aproximación de los sistemas judiciales y procesales europeo y nacional aparece en la sentencia XC y otros, en la evaluación de la compatibilidad del derecho austriaco con el principio de efectividad. En este caso, se puede considerar la expresión de un respeto hacia las normas nacionales que aseguran la firmeza de las resoluciones judiciales puesto que aparece

24 La frase aparece por primera vez en la sentencia del Tribunal de Justicia de 16 de marzo de 2006, Kapferer, C-234/04, EU:C:2006:178, apdo. 20. Aparece por ejemplo en las sentencias del Tribunal de Justicia de 19 de abril de 2012, Artegodan GmbH, C-221/10 P, EU:C:2012:216, apdo. 86; de 24 de enero de 2013, Comisión/España, C-529/09, EU:C:2013:31, apdo. 64; o del Tribunal General, 5 de julio de 2012, Grecia/Comisión, T-86/08, EU:T:2012:345, apdo. 33. Por ejemplo, en la sentencia del Tribunal de Justicia Kapferer (cit. supra), apdo. 20, o en el Auto del Tribunal de la Función Pública de 7 de junio de 2016, Marco Verile. Comisión, F-108/12, EU:F:2016:125, apdo. 38.

26 Sobre esta cuestión me refiero en particular a la posición expresada por el juez Tizzano en Tizzano y Gencarelli (2011).

27 Por ejemplo, su utilización en la sentencia Kapferer aparece como un elemento más en su intento de tranquilizar a los jueces nacionales acerca de la incidencia que podría tener la sentencia Kühne and Heitz.

Las sentencias Artegodan y Comisión/España tratan del derecho procesal de la Unión. 
en una línea argumentativa que rechaza toda obligación de extender al ámbito del derecho de la Unión un procedimiento que es una excepción a la cosa juzgada. Tras haber reiterado la regla según la cual el derecho de la Unión no exige la creación de vías de recurso específicas ante los tribunales nacionales ${ }^{29}$, el Tribunal recuerda su jurisprudencia Kapferer y la frase relativa a la importancia de la cosa juzgada en los ordenamientos jurídicos de la UE y de los Estados miembros, e incluye una referencia indirecta a la seguridad jurídica como fundamento común de este principio en todo sistema judicial ${ }^{30}$. Las otras sentencias citadas son todas relativas a la cosa juzgada, como principio de derecho nacional ${ }^{31}$ o de derecho de la Unión ${ }^{32}$.

En la jurisprudencia Kapferer, como en otras sentencias, la asimilación de la justificación y de la protección de la cosa juzgada en los ordenamientos jurídicos nacionales y en el de la Unión sirve para tranquilizar a los jueces nacionales, convenciéndoles de que la limitación de la autonomía procesal no puede llegar hasta poner en tela de juicio la cosa juzgada. El Tribunal se apoya en su jurisprudencia anterior para establecer la base de su razonamiento acerca de la efectividad: la importancia de la cosa juzgada significa que el derecho de la Unión no puede obligar al juez nacional a descartar la aplicación de normas que la garantizan. De ahí que, como ya había indicado el Tribunal en sentencias anteriores, el derecho de la Unión no exija que se subsane sistemáticamente toda situación nacional incompatible con él, mediante la revisión de una resolución judicial firme ${ }^{33}$.

El hecho de recordar la jurisprudencia bien establecida en lo que concierne a la importancia de la cosa juzgada y al respeto por el juez de la Unión de las normas de derecho nacional que permiten su aplicación no solo aparece como un automatismo por parte del Tribunal de Justicia. Las referencias a la jurisprudencia anterior permiten asentar otros aspectos del razonamiento,

29 Apdo. 51 de la sentencia $X C$ y otros, que se refiere a la Sentencia del Tribunal de Justicia de 13 de marzo de 2007, Unibet, C-432/05, EU:C:2007:163. En esta sentencia el Tribunal de Justicia indica que el derecho de la Unión, y en particular el principio de tutela judicial efectiva de los derechos conferidos a los justiciables por el ordenamiento jurídico de la Unión, no exige que los Estados miembros establezcan acciones autónomas que tengan por objeto, con carácter principal, el examen de la compatibilidad de disposiciones nacionales con normas de derecho de la Unión.

30 Apdo. 52 de la sentencia XC y otros.

31 Sentencias de 10 de julio de 2014, Pizzarotti, C-213/13, EU:C:2014:2067; y de 6 de octubre de 2015, Târ ia, C-69/14, EC:C:2015:662.

32 Sentenciasde29dejuniode2010, Comisión/Luxemburgo,C-526/08,EU:C:2010:379; y de 29 de marzo de 2011, ThyssenKrupp, C-352/09 P, EU:C:2011:191.

33 Sentencia XC y otros, apdos. 53-54. 
como lo demuestra la estructura de las conclusiones del abogado general ${ }^{13}$. En efecto, el hecho de que el derecho de la UE respete no solamente la autonomía procesal de los Estados miembros sino específicamente la importancia de las normas relativas a la cosa juzgada es una primera etapa esencial en la prueba de la inexistencia de una violación del principio de efectividad del derecho de la Unión. Como lo expresan claramente las conclusiones, existe evidentemente una tensión entre respeto de la cosa juzgada nacional y efectividad del derecho de la Unión ${ }^{35}$. Así pues, comenzar por la reafirmación de la importancia del primer principio permite evaluar la limitación de la efectividad de manera más clemente.

\section{LAS LIMITACIONES SUFICIENTES A LA COSA JUZGADA NACIONAL EN EL DERECHO DE LA UE}

El Tribunal de Justicia, al igual que su abogado general, insiste en la importancia de la cosa juzgada como principio en los ordenamientos jurídicos de la UE y de los Estados miembros porque esta afirmación sirve luego de base para evaluar la aceptabilidad de la restricción a la efectividad del derecho de la Unión que causa la imposibilidad de revisar sentencias firmes. La tensión puesta de manifiesto por el abogado general Saugmandsgaard Øe es inevitable, puesto que la firmeza de las sentencias impide que algunas de ellas, en las cuales luego se identifica una mala interpretación o aplicación del derecho, sean rectificadas. Esto no es un problema específico del derecho de la Unión. Las normas procesales relativas a la cosa juzgada son la expresión de compromisos entre la búsqueda de la sentencia más justa posible, basada en la realidad de los hechos y del derecho aplicable, y la necesaria protección de la función judicial en el ordenamiento jurídico, de la estabilidad de las relaciones jurídicas establecidas en la sentencia y del derecho a una tutela judicial efectiva de los justiciables que puede incluir un derecho a revisar resoluciones cuando aparecen nuevos elementos. La firmeza que adquieren las resoluciones judiciales se justifica por estos últimos motivos a pesar de que impide que se persigan indefinidamente la búsqueda de la verdad y de la justicia perfecta en

\footnotetext{
34 En la parte relativa a la inexistencia de una violación del principio de efectividad, el abogado general comienza por una primera parte titulada «El principio: respeto a la autoridad de cosa juzgada de las resoluciones nacionales» (puntos 43 y ss. de las conclusiones).

35 Punto 45 de las conclusiones.
} 
el juicio ${ }^{36}$. Los mismos motivos justifican el respeto dado a la cosa juzgada, incluso en el escalón nacional, aunque impidan modificar una sentencia claramente incompatible con el derecho de la Unión.

La efectividad no es un principio absoluto y no se puede impedir cualquier vulneración de esta. Otros principios, basados en componentes tan fundamentales del ordenamiento jurídico como la seguridad jurídica o la tutela judicial efectiva, pueden justificar una efectividad menor. Así como lo recuerda el abogado general, una reducción de la efectividad del derecho de la Unión no implica por sí misma una violación de este principio ${ }^{37}$. Por esta razón la jurisprudencia del Tribunal no exige que se reconsideren sistemáticamente las resoluciones nacionales anteriores incompatibles con sus propias sentencias ${ }^{38}$. Por lo demás, el derecho de la Unión ya prevé mecanismos que permiten limitar la incidencia de la cosa juzgada nacional en su efectividad: por una parte, estableciendo excepciones a las normas nacionales en cuestión, por otra, con el principio de responsabilidad del Estado.

El Tribunal no menciona las excepciones que ha impuesto a la cosa juzgada nacional en esta sentencia. El abogado general lo había hecho, y había observado correctamente que aquellas excepciones al respeto de las normas nacionales que protegen la cosa juzgada correspondían a situaciones muy particulares, en las cuales características estructurales del procedimiento nacional hacían imposible o excesivamente difícil el ejercicio de los derechos conferidos por el derecho de la Unión ${ }^{39}$. Por ejemplo, en el caso de las reglas italianas relativas a la cosa juzgada en materia fiscal, que dieron lugar a los casos Fallimento Olimpiclub ${ }^{40}$ y Lucchini $^{41}$, las decisiones tomadas por el Tribunal se deben probablemente al área de competencia en cuestión (el I.V.A., o sea un área de competencia exclusiva para la Unión) y al alcance temporal inhabitual dado a la cosa juzgada en tales situaciones. El derecho italiano hubiera impedido toda rectificación de una aplicación incorrecta del derecho de la Unión en años fiscales posteriores y, por lo tanto, toda rectificación de la situación fiscal de los contribuyentes afectados y toda recuperación de las sumas pendientes

36 El proceso no puede tener como único objetivo la búsqueda de la verdad porque sería una labor imposible e interminable. La firmeza de las resoluciones judiciales no se justifica por el hecho de que el juez haya identificado la verdad, sino por la necesidad de dar una conclusión al procedimiento judicial. Véase Tomasin (1975: 330).

37 Puntos 45-46 de las conclusiones.

38 Apdo. 54 de la sentencia.

39 Puntos 58 y ss. de las conclusiones.

40 Sentencia de 3 de septiembre de 2009, Fallimento Olimpiclub Srl, C-2/08, EU:C:2009:506.

41 Véase supra, nota 21. 
(Tizzano y Gencarelli, 2011: 277-278). Estas circunstancias justificaban, desde el punto de vista del Tribunal de Justicia, que una excepción no se debe asimilar a una voluntad de dificultar la plena aplicación de las reglas relativas a la cosa juzgada en los Estados miembros. Una explicación similar vale para la sentencia Asturcom acerca de laudos arbitrales en España ${ }^{42}$. Estas excepciones no son aplicables en este caso ${ }^{43}$, pero son una primera prueba de la ausencia de necesidad de extensión del ámbito del art. 363a. El derecho de la Unión prevé excepciones a la cosa juzgada nacional cuando parecen indispensables al Tribunal de Justicia.

El principio de la responsabilidad del Estado miembro derivada de una resolución de un órgano jurisdiccional, o principio Köbler ${ }^{44}$, derivado del principio Francovich ${ }^{45}$, es otro instrumento que permite al Tribunal limitar el impacto de una resolución judicial sobre la efectividad del derecho de la Unión. Cabe señalar que, aunque el Tribunal de Justicia lo haya negado ${ }^{46}$, este principio vulnera indirectamente la cosa juzgada dado que pone en tela de juicio la resolución que ha causado la violación del derecho de la Unión ${ }^{47}$. Sin embargo, es cierto que permite proteger los derechos conferidos a los particulares sin afectar directamente a la firmeza de la resolución judicial nacional. De ahí que el Tribunal de Justicia, como su abogado general, se refiera a este principio como a una prueba de la ausencia de necesidad de acceder a la vía de recurso establecida en el art. 363a ${ }^{48}$ para garantizar la efectividad del derecho de la Unión. Aunque exista una resolución judicial firme contraria al derecho de la Unión, este principio debe permitir que un justiciable obtenga reparación y contribuir a desalentar las violaciones del derecho de la Unión por parte de órganos judiciales.

El razonamiento desarrollado por el Tribunal de Justicia y su abogado general se apoya en el estatus de la cosa juzgada nacional en el derecho de la Unión para demostrar que la efectividad de este derecho no requiere mecanismos tales como el del art. 363a. Por una parte, por lo general el principio

42 Punto 60 de conclusiones y nota 34: sentencias del Tribunal de Justicia de 6 de octubre de 2009, Asturcom, C-40/08, EU:C:2009:615; y de 26 de octubre de 2006, Mostaza Claro, C-168/05, EU:C:2006:675.

Apdos. 55-56 de la sentencia, punto 62 de las conclusiones: nada indica que en este caso la efectividad del derecho de la Unión esté tan gravemente vulnerada como para justificar una inaplicación del derecho procesal austriaco.

44 Véase supra, nota 20.

45 Sentencia de 19 de noviembre de 1991, Francovich, C-6 y 9/90, EU:C:1991:428.

46 Apdo. 39 de la sentencia Köbler.

47 Entre las numerosas reacciones a la sentencia Köbler y a esta jurisprudencia, conviene señalar a Adinolfi (2012: 287) y a Wattel (2004).

48 Apdo. 58 de la sentencia, punto 56 de las conclusiones. 
es el respeto del derecho procesal de los Estados miembros en lo que se refiere a la cosa juzgada, aunque ello suponga limitar la efectividad del derecho de la Unión. Por otra parte, el derecho de la Unión ya contiene mecanismos que permiten contener los efectos adversos de la cosa juzgada nacional, que se deben utilizar en casos en los que existe un riesgo de vulneración grave de la efectividad del derecho de la Unión.

\section{UNA EXPOSICIÓN DE LAS DIFERENCIAS ESENCIALES ENTRE LA EFECTIVIDAD DE LOS SISTEMAS JURISDICCIONALES DE LA UNIÓN EUROPEA Y DEL CEDH}

La necesidad de respetar la autonomía procesal de los Estados miembros en lo que se refiere a la cosa juzgada y a la firmeza de las resoluciones judiciales es solo un aspecto de la motivación de esta sentencia. La cuestión de la necesidad de extender el ámbito de vías de recurso tales como la del art. 363a al derecho de la Unión no solamente pone en tela de juicio el balance necesario entre efectividad y cosa juzgada. También, y de manera quizás más fundamental, interroga al Tribunal de Justicia sobre las características generales del sistema jurisdiccional de la UE. Estas vías de recurso han sido introducidas porque se consideran necesarias para asegurar la efectividad del CEDH y de las sentencias del TEDH. Para ofrecer una respuesta al Oberster Gerichsthof, había que establecer una comparación entre los sistemas jurisdiccionales de la Unión y del $\mathrm{CEDH}$, con el objetivo de determinar si el mismo razonamiento era aplicable en la Unión. Por lo demás, esta comparación era necesaria para determinar si el ámbito actual de la vía de recurso era compatible con el principio de equivalencia. El Tribunal de Justicia establece las diferencias fundamentales entre los dos sistemas para justificar la respuesta dada al tribunal nacional, según la cual los principios de efectividad y de equivalencia no requieren la extensión del mecanismo previsto en el art. 363a al derecho de la Unión. Para ello demuestra, en primer lugar, que este tipo de vía de recurso tiene un vínculo consustancial con las características del sistema establecido por el CEDH (1) y, en segundo lugar, que el test de equivalencia no es pertinente debido a las diferencias con el sistema de la UE (2).

\section{LA JUSTIFICACIÓN DEL PROCEDIMIENTO DE REVISIÓN POR LAS CARACTERÍSTICAS ESPECÍFICAS DEL SISTEMA JURISDICCIONAL DEL CEDH}

La comparación entre un recurso tal como el que establece el art. 363a, basado en el CEDH, y los recursos que permiten la aplicación efectiva del 
derecho de la UE es necesaria para determinar la compatibilidad de esta norma de derecho procesal nacional con el principio de cooperación leal. Según una jurisprudencia reiterada del Tribunal de Justicia, el principio de equivalencia exige que la regulación procesal de los recursos que permiten salvaguardar los derechos que el derecho de la Unión confiere a los justiciables no sea menos favorable que la de recursos similares que se apoyan en normas de derecho interno ${ }^{49}$. El Tribunal de Justicia y el abogado general recuerdan este estándar ${ }^{50}$ y llegan a la misma conclusión, según la cual el recurso regulado en el art. 363a debe considerarse un recurso de derecho interno, pero no puede considerarse «similar» a los que permiten proteger el derecho de la Unión, o más precisamente los derechos fundamentales que este consagra ${ }^{51}$. Los razonamientos son algo distintos, pero se corresponden en lo esencial: la diferencia se debe a que un recurso como el que establece el art. 363a forma parte del sistema del CEDH.

El abogado general Saugmandsgaard Øe rechaza en principio toda equiparación de tales recursos con recursos de derecho interno en el sentido del principio de equivalencia. Según él, este tipo de mecanismo no tiene por objetivo permitir la aplicación de normas de derecho interno, puesto que está basado en el $\mathrm{CEDH}^{52}$. Por tanto, no se trata de establecer un recurso más favorable para los justiciables si eligen basar su acción en normas de derecho interno y el principio de equivalencia no es pertinente. Este razonamiento es convincente si se tiene en cuenta, como lo hace el abogado general, la función de este principio. Es cierto que su razón de ser en la jurisprudencia es permitir al Tribunal evitar tratos discriminatorios del derecho de la Unión en relación con el derecho interno, impidiendo que las condiciones en las cuales se desarrollan los procedimientos sean menos favorables si los justiciables eligen apoyarse en el derecho de la Unión ${ }^{53}$. Los mecanismos de revisión, como el art. 363a, no permiten recursos basados en el derecho interno y por lo tanto no pueden causar este tipo de "proteccionismo procesal " $^{54}$.

Sin embargo, en un sentido más formal, este recurso destinado a obtener la repetición del procedimiento penal es un recurso de derecho interno y no, estrictamente hablando, de derecho internacional. Por esta razón, el tribunal

\footnotetext{
49 Sentencia de 16 de diciembre de 1976, Rewe, 33/76, EU:C:1976:188, apdo. 5.

50 Apdo. 25 de la sentencia, punto 65 de las conclusiones.

51 Apdo. 35 de la sentencia, punto 70 de las conclusiones.

52 Punto 67 de las conclusiones.

53 Punto 68 de las conclusiones.

54 Según la expresión empleada por el abogado general en el punto 68 de las conclusiones.
} 
remitente, el Gobierno austríaco y el Tribunal de Justicia no dudan de la aplicabilidad del principio de equivalencia ${ }^{55}$ y el mismo abogado general se concentra $^{56}$ en la cuestión central, que es la del carácter «similar» de este recurso con un recurso destinado a proteger el derecho de la UE. En principio, un recurso que se apoya en un derecho fundamental enunciado en el Convenio parece similar a un recurso basado en el mismo derecho fundamental, enunciado en la Carta de los Derechos Fundamentales o en otro instrumento de derecho de la Unión. Es evidente en la solicitud de los interesados en el procedimiento nacional, que alegan que existe una violación de derechos que les han sido reconocidos por el CEDH y por el derecho de la Unión. El art. 52, apdo. 3, de la Carta fomenta este tipo de planteamiento (Peers y Prechal, 2014: 1490 y ss.). La presunción de equivalencia ${ }^{57}$ alienta la percepción de los sistemas europeos de protección de los derechos fundamentales como un conjunto lo más coherente posible, con niveles de protección similares y, por lo tanto, se alienta la utilización combinada (o bien alternativa) de diferentes instrumentos. ¿Por qué no considerar un recurso basado en el CEDH como similar a un recurso basado en la Carta?

No obstante la presunta equivalencia en el contenido de los derechos enunciados en los dos ordenamientos europeos, el principio de equivalencia supone examinar la naturaleza de los recursos y no solamente la de las normas que permiten proteger. Así pues, según la jurisprudencia del Tribunal de Justicia, hay que examinar «el objeto, la causa y los elementos esenciales» del recurso de derecho interno para determinar si es similar a un recurso de derecho de la Unión. Tanto el Tribunal de Justicia como su abogado general se focalizan en la función de este tipo de recurso destinado a obtener la repetición del procedimiento penal, y en su relación con las características del sistema jurisdiccional en el que se apoya el CEDH. Tal como señala el Tribunal, la justificación de esta vía de recurso extraordinaria se encuentra en la propia naturaleza del Convenio, razón por la cual mantiene un estrecho vínculo funcional con el procedimiento ante el $\mathrm{TEDH}^{58}$. Por la misma razón, la respuesta al tribunal remitente debe ser que no es un recurso similar a un recurso destinado a proteger el derecho de la Unión.

El vínculo de este tipo de recurso con la naturaleza del funcionamiento del sistema jurisdiccional establecido en el CEDH es evidente. La exigencia

\footnotetext{
55 Apdo. 26 de la sentencia.

56 La discusión de la noción de «recurso de derecho interno» solo ocupa tres apdos.

57 Sentencia del TEDH de 30 de junio de 2005, Bosphorus Airlines, n. ${ }^{\circ}$ 45036/98, apdos. 155 y 156.

58 Apdos. 29-31 de la sentencia.
} 
según la cual no se puede recurrir al TEDH sino después de agotar las vías de recursos nacionales ${ }^{59}$ implica, en efecto, la existencia de una resolución firme en el nivel nacional, dado que supone la existencia de una resolución en última instancia, que adquiere automáticamente fuerza de cosa juzgada ${ }^{60}$. Por esta razón, en principio, el TEDH no puede pronunciarse sobre una situación nacional antes de que exista una resolución judicial firme, y la efectividad de sus sentencias siempre depende de las acciones tomadas por el Estado después de que los interesados hayan agotado los recursos del derecho interno ${ }^{61}$. Esta regla de admisibilidad se justifica, en primer lugar, por la necesidad de permitir al Estado que rectifique él mismo la violación, mediante sus jurisdicciones, $\mathrm{y}$, en segundo lugar, por la de limitar el número de casos que lleguen a ser tratados por el TEDH. Sin embargo, este sistema desfavorece claramente a las víctimas de la violación que han interpuesto el recurso ante el TEDH, puesto que no se prevé en el Convenio ninguna obligación de modificar la resolución que cristaliza la violación, por lo que su situación jurídica se mantiene. Los límites de este sistema han sido claramente identificados desde hace tiempo y el Protocolo n. ${ }^{\circ} 16$ debe permitir un acceso al TEDH antes de que exista una resolución nacional firme ${ }^{62}$, pero las reglas establecidas en el mismo le darán una eficacia limitada en este respecto, como bien señala el abogado general ${ }^{63}$.

Esta falta de efectividad de las sentencias del TEDH para las víctimas individuales de las violaciones de derechos fundamentales ha conducido de manera consecuente a varios Estados miembros a establecer vías de recursos extraordinarias para permitir la revisión de resoluciones firmes, particularmente en materia penal. El art. 363a es un ejemplo de este tipo de mecanismo, que solo se puede utilizar cuando ya existe una resolución judicial firme y el TEDH ha apreciado una violación, o cuando se anticipa tal apreciación. Este recurso debe, pues, considerarse una solución aplicable en casos extraordinarios en los cuales parece indispensable dar eficacia a la

59 Art. 35, apdo. 1, del CEDH.

60 Apdo. 32 de la sentencia.

61 Punto 76 de las conclusiones.

62 El Protocolo n. ${ }^{\circ} 16$ permite a los más altos órganos jurisdiccionales de un Estado miembro solicitar la opinión del TEDH sobre la interpretación o la aplicación de derechos y libertades enunciados en el Convenio o sus protocolos. Esta solicitud se debe hacer en el marco de un asunto pendiente. El primer dictamen del TEDH en la aplicación de este protocolo fue emitido el 10 de abril de 2019 (n. ${ }^{\circ}$ P16-2018-001), tras una remisión hecha por la Cour de cassation francesa en un asunto relacionado con la maternidad de alquiler.

63 Apdos. 80-82 de las conclusiones. 
sentencia (actual o esperada) del TEDH en el caso individual del interesado y no mantener esta violación del derecho fundamental en el ordenamiento jurídico. En otras palabras, es un mecanismo cuya existencia depende de la presuposición de que una sentencia del TEDH no puede producir efectos directos sobre la situación del individuo que ha sufrido la violación, y busca remediar esta falta de eficacia en algunos casos especialmente graves. Si fuera posible asegurar la plena aplicación del CEDH de otra forma, por ejemplo obteniendo una sentencia del TEDH antes de que existiese una resolución judicial firme a nivel nacional, tales excepciones a la cosa juzgada no serían necesarias.

Esta es justamente la diferencia entre el sistema establecido por el Convenio y el ordenamiento jurídico de la Unión que señala el Tribunal de Justicia, y en la que se basa para descartar la prueba de equivalencia. Puesto que la efectividad de la Unión no depende de tales mecanismos y como su integración en los sistemas judiciales nacionales se lleva a cabo de forma muy diferente, el recurso establecido en el art. 363a no puede considerarse «similar» a ningún recurso de los que permite aplicar el derecho de la Unión. Siguiendo una práctica frecuente desde hace algunos años, el Tribunal de Justicia fundamenta esta respuesta en una argumentación que no solo se basa en la relación que existe entre los mecanismos tales como el del art. 363a, sino que también enfatiza el marco constitucional de la $\mathrm{UE}^{64}$.

\section{LAS DIFERENCIAS FUNDAMENTALES ENTRE EL SISTEMA JURISDICCIONAL DE LA UE Y EL DEL CEDH}

La principal función del principio de equivalencia en el marco de la autonomía procesal de los Estados miembros es evitar que se establezcan condiciones menos favorables para acciones basadas en el derecho de la Unión en comparación con las que se apoyan en el derecho interno. De este modo, completa el principio de eficacia para garantizar que los justiciables siempre puedan útilmente reclamar los derechos que derivan del derecho de la Unión en el sistema jurisdiccional nacional. La cuestión prejudicial en este caso interroga al Tribunal de Justicia sobre la necesidad de extender un mecanismo introducido específicamente para asegurar la efectividad del $\mathrm{CEDH}$ al derecho de la Unión. Exponiendo los motivos por los cuales este tipo de mecanismo no se debe extender demasiado, debido a la importancia de la cosa

64 Expresión que no se encuentra explícitamente en la sentencia pero sí es empleada por el abogado general Saugmandsgaard Øe en el punto 5 de sus conclusiones, en referencia al Dictamen 2/13 (véase supra nota 18). 
juzgada, el Tribunal de Justicia demuestra también esforzarse en demostrar por qué no es necesario en el ámbito del derecho de la Unión. Si la necesidad del recurso establecido en el art. 363a reside en las deficiencias del sistema jurisdiccional del CEDH, el de la Unión permite, según el Tribunal de Justicia y su abogado general, un nivel de efectividad suficiente como para evitar tales vías de recursos extraordinarias.

Por una parte, el Tribunal de Justicia demuestra que las limitaciones impuestas por su propia jurisprudencia a la cosa juzgada nacional ya permiten un nivel de efectividad suficiente del derecho de la Unión, teniendo en cuenta el respeto necesario para la firmeza de las resoluciones judiciales nacionales en el sistema jurisdiccional de la Unión. Por otra parte, dedica una parte importante de la sentencia a demostrar que las características generales de este sistema jurisdiccional aseguran la efectividad del derecho de la Unión y la protección de las víctimas de violaciones en los Estados miembros. Las características esenciales del sistema jurisdiccional de la Unión forman parte del marco constitucional de la UE, en el que ocupan una función imprescindible. Su importancia en la visión que desarrolla el Tribunal de Justicia de su propio ordenamiento jurídico es evidente en la jurisprudencia de los últimos años, en la que protege con denuedo la estructura de este sistema y la naturaleza de sus relaciones con los jueces nacionales.

La primacía y el efecto directo son citados por el abogado general como las primeras características del marco constitucional de la UE que aseguran la efectividad de este derecho sin obligar a los Estados a poner en entredicho la cosa juzgada ${ }^{65}$. Es cierto que estos principios fundamentales del derecho de la Unión deben permitir la plena aplicación de este derecho en los tribunales nacionales, aunque sea descartando una disposición contraria del derecho nacional. Sin embargo, es posible considerar que, por su naturaleza misma o debido a la influencia del derecho de la Unión, estas características se están extendiendo al CEDH (Martinico, 2012: 407 y ss.; Bourgeois, 2016: 21-23). Según el TEDH, no cabe duda de que el Convenio tiene primacía sobre toda norma de derecho interno ${ }^{66}$. La primacía del CEDH sobre la legislación nacional depende sin duda del marco constitucional de cada Estado, pero los derechos fundamentales reconocidos en el Convenio existen también como normas constitucionales en la mayoría de estos sistemas, normas cuya interpretación puede ser influenciada por la del Convenio. En lo que se refiere al efecto directo, si bien es cierto que no aparece en la jurisprudencia del TEDH con

65 Apdos. 50-52 de las conclusiones.

66 Sentencia del TEDH de 29 de octubre de 1992, Open Door, n. ${ }^{\circ} 14234 / 88$ y n. ${ }^{\circ}$ $14235 / 88$. 
tanta firmeza como en la del Tribunal de Justicia, los derechos protegidos por el CEDH son directamente aplicables en varios Estados miembros (Sudre, 2016: 24-25) e integrados en el derecho interno en otros con una posición diferente a la de otros instrumentos legislativos ${ }^{67}$. Los estándares europeos establecidos por el TEDH deben prevalecer erga omnes y conducir a los Estados miembros a modificar sus ordenamientos jurídicos aunque no estén directamente afectados por una sentencia ${ }^{68}$.

La diferencia fundamental entre CEDH y Unión Europea no reside en la afirmación, hecha por el Tribunal de Justicia, de la primacía o del efecto directo de las normas que componen su ordenamiento jurídico en los Estados miembros, sino en la capacidad del sistema jurisdiccional construido alrededor de estas normas de imponer el acatamiento de estos principios. Como recuerda a menudo el Tribunal de Justicia, la eficacia del derecho de la Unión se debe a la integración de los órganos jurisdiccionales nacionales en el sistema jurisdiccional de la UE. Esta integración se debe en gran parte al mecanismo de la cuestión prejudicial, por lo que no sorprende que el Tribunal defienda este mecanismo en particular, considerándolo "la piedra angular del sistema jurisdiccional» de la Unión ${ }^{69}$. Este mecanismo permite establecer un diálogo entre jueces nacionales y europeos acerca de la interpretación correcta y del contenido del derecho de la Unión incluso antes de que se haya tomado una sola resolución judicial en el asunto. De esta manera, en los casos en los cuales los jueces remiten una cuestión prejudicial al Tribunal se puede esperar que la plena efectividad del derecho de la Unión sea garantizada por el sistema judicial nacional mismo. Así pues, el Tribunal de Justicia y su abogado general recuerdan que el procedimiento de remisión prejudicial se puede utilizar en cualquier fase del procedimiento nacional ${ }^{70}$, y es obligatorio, según el apdo. 3

67 Por ejemplo, con el Human Rights Act de 1998 en Reino Unido, que da valor legislativo al CEDH pero incluye una obligación de tomar en cuenta la jurisprudencia del TEDH.

68 El efecto erga omnes de sus propias sentencias ha sido establecido por el TEDH desde su Sentencia de 18 de enero de 1978, Irlanda c. Reino Unido, n. ${ }^{\circ}$ 5310/71.

69 Apdo. 41 de la sentencia $X C$ y otros. La expresión es empleada con frecuencia por el Tribunal de Justicia acerca del procedimiento, por ejemplo en el Auto del presidente del Tribunal de Justicia de 15 de noviembre de 2018, Comisión/Polonia, C-619/18, EU:C:2018:910, apartado 22.

70 Apdo. 42 de la Sentencia XC y otros. La sentencia se refiere a la Sentencia de 5 de julio de 2016, Ognyanov, C-614/14, EU:C:2016:514, pero la libertad de los jueces nacionales de optar por una remisión prejudicial en cualquier momento está bien establecida en la jurisprudencia, incluso en casos en los cuales el Tribunal de Justicia ya 
del art. 267 TFUE, cuando el asunto se presenta ante un órgano jurisdiccional cuya decisión no admite recurso ${ }^{71}$.

El procedimiento de la remisión prejudicial establece además una relación particular entre tribunales y permite concebir un sistema jurisdiccional único, que comprende a la vez los tribunales de la Unión y los órganos nacionales (Weatherill, 2016: 154). El art. $19 \mathrm{TUE}^{72}$, que obliga a los Estados miembros a contribuir a la tutela judicial efectiva en los ámbitos cubiertos por el derecho de la Unión, y la interpretación de esta disposición por el Tribunal de Justicia y el Tribunal General, según la cual este artículo es la manifestación de un sistema jurisdiccional común, no tendrían mucho sentido sin el procedimiento de remisión prejudicial. Conviene destacar la importancia que el Tribunal de Justicia ha dado a este artículo en sus sentencias recientes para justificar una transición desde la presunción de respeto a los derechos humanos en los Estados miembros hasta el establecimiento de una obligación de respeto, bajo la supervisión del mismo Tribunal de Justicia, para asegurar la sostenibilidad del sistema jurisdiccional de la $\mathrm{UE}^{73} \mathrm{y}$, en particular, de la cooperación judicial en materia penal ${ }^{74}$. Este procedimiento permite considerar a los jueces nacionales como miembros de un sistema jurisdiccional

ha respondido a una primera remisión: Sentencia de 27 de marzo de 1963, Da Costa, 28-30/62, EU:C:1963:6.

71 Apdo. 43 de la sentencia XC y otros y punto 54 de las conclusiones. La sentencia se refiere en particular a la Sentencia del Tribunal de Justicia de 9 de septiembre de 2015, Ferreira da Silva, C-160/14, EU:C:2015:565, pero aquí también la jurisprudencia está bien establecida. La sentencia Da Costa (véase supra) o la Sentencia de 6 de octubre de 1982, CILFIT, 283/81, EU:C: 1982:335. La misma obligación existe cuando existen dudas acerca de la validez de la disposición de derecho de la Unión que se debe aplicar en un asunto: Sentencia del 22 de octubre de 1987, Foto-Frost, 314/85, EU:C:1987:452.

72 «El Tribunal de Justicia de la Unión Europea comprenderá el Tribunal de Justicia, el Tribunal General y los tribunales especializados. Garantizará el respeto del Derecho en la interpretación y aplicación de los Tratados.

Los Estados miembros establecerán las vías de recurso necesarias para garantizar la tutela judicial efectiva en los ámbitos cubiertos por el Derecho de la Unión».

73 Sentencias del Tribunal de Justicia de 27 de febrero de 2018, Associação Sindical dos Juízes Portugueses, C-64/16, EU:C:2018:117, apdos. 29-37; y de 7 de febrero de 2019, Escribano Vindel, C 49/18, EU:C:2019:106, apdos. 62-73.

74 Sentencia del Tribunal de Justicia de 25 de julio de 2018, Minister for Justice and Equality (LM), C-216/18 PPU, EU:C:2018:586, apdos. 50-51; y Conclusiones del Abogado general Tanchev presentadas el 11 de abril de 2019, Comisión c. Polonia, C-619/18, EU:C:2019:325, puntos 61 ss. 
europeo y como «Jueces comunitarios de Derecho común» ${ }^{75}$, y por ello las obligaciones de estos mismos jueces de garantizar la tutela judicial efectiva de los derechos que los justiciables derivan del derecho de la Unión adquieren un aspecto diferente ${ }^{76}$.

La amenaza que constituye la posibilidad de recursos por incumplimiento basados en el art. 258 TFUE por infracciones cometidas por resoluciones judiciales ${ }^{77}$ tal vez no sea muy diferente de la de recursos similares ante el TEDH. En los dos sistemas, por lo general, las normas derivadas de los tratados europeos deben ser aplicadas a nivel nacional sin necesidad de acceder a un tribunal europeo. Sin embargo, en el sistema jurisdiccional de la Unión el recurso por incumplimiento causado por un tribunal nacional debe considerarse una excepción no solo a la aplicación habitual del derecho europeo por los jueces nacionales sino también en el contexto de las diferentes vías de recurso que existen ante el Tribunal de Justicia de la Unión Europea y de la relación privilegiada entre los jueces nacionales y el Tribunal de Justicia: la remisión prejudicial. El mecanismo que establece el art. 267 TFUE debe ser el modo privilegiado de acceso del individuo a la interpretación del derecho de la Unión que ofrece el Tribunal de Justicia, sin necesidad de esperar a una resolución judicial nacional. Si bien es cierto que esta descripción del sistema jurisdiccional de la Unión Europea no se corresponde con la realidad y que a menudo los jueces de primera instancia no remiten cuestiones prejudiciales o los tribunales supremos muestran reticencias a emplear este mecanismo ${ }^{78}$,

75 Según la expresión consagrada en la Sentencia del Tribunal de Primera Instancia del 10 de julio de 1990, Tetra Pak, T-51/89, EU:T:1990:41, apdo. 42.

76 No es necesario ni posible recordar aquí los numerosos estudios acerca de la importancia de la remisión prejudicial y de la relación que establece entre el juez nacional y el Tribunal de Justicia en el derecho procesal de la Unión Europea. Recordemos simplemente algunas contribuciones notables: Barav (1993); Lagrange (1974); Lenaerts et al. (2014: 51); Picod (2002: 199-232); Weatherill (2016: 154-159).

77 La evolución hacia el pleno reconocimiento de la posibilidad de incoar un procedimiento de infracción contra un Estado miembro debido a la acción (o inacción) de sus órganos jurisdiccionales pone de manifiesto la importancia que da el Tribunal de Justicia a la remisión prejudicial: sentencias de 9 de diciembre de 2003, Comisión c/ Italia, C-129/00, EU:C:2003:656; de 12 de noviembre de 2009, Comisión/ España, C-154/08, EU:C:2009:695; y de 4 de octubre de 2018, Comisión/Francia, C-416/17, EU:C:2018:811.

78 El caso que dio lugar a la sentencia del Tribunal de Justicia del 4 de octubre de 2018, Comisión cl Francia (véase supra) es un buen ejemplo de este tipo de dificultad. Los jueces franceses de lo Administrativo de primera instancia y de segunda instancia no suelen remitir cuestiones prejudiciales y no lo hicieron en este caso, aunque 
el sistema funciona lo suficientemente bien como para dar una connotación muy diferente a los principios de efecto directo y de primacía en el derecho de la Unión.

En comparación con el que ha establecido el CEDH, el sistema jurisdiccional de la UE tiene una ventaja significativa en lo que concierne a la efectividad de la protección del justiciable. En principio, este sistema contiene elementos que facilitan grandemente la obtención de la tutela efectiva de los derechos que una persona deriva del derecho de la Unión sin que se produzca una resolución nacional firme. Este es el motivo por el cual no parece necesario introducir excepciones a la cosa juzgada con vías de recurso extraordinarias que permitirían tener en cuenta una sentencia del Tribunal de Justicia dictada después de una resolución nacional. Así pues, los componentes jurisdiccionales del marco constitucional de la Unión significan que la cuestión de la equivalencia entre procedimientos tales como el que establece el art. 363a y los que permiten proteger el derecho de la Unión no es pertinente. No existen procedimientos similares en el ámbito del derecho de la UE porque no son necesarios. Tal vez podrían ser útiles, si el objetivo fuera alcanzar la efectividad máxima de las sentencias del Tribunal de Justicia, pero este no es el objetivo ya que otras consideraciones de igual importancia exigen el respeto de la cosa juzgada.

\section{CONCLUSIÓN}

La respuesta que el Tribunal de Justicia ofrece a la cuestión prejudicial del Oberster Gerichsthof no debe sorprender. A pesar de lo que la reacción algo excesiva de algunos autores a algunas excepciones podía dejar pensar, la jurisprudencia del Tribunal de Justicia ha sido bastante constante en su respeto hacia las reglas nacionales que protegen la cosa juzgada. Ha ido siempre más allá de lo que exige la autonomía procesal tratando de asimilar la posición y la importancia de la cosa juzgada en el derecho interno y en el derecho de la Unión. De este modo, el Tribunal de Justicia intenta consolidar el sistema

habían identificado problemas en la interpretación del derecho de la Unión. El Conseild'État tampoco respetó del todo su obligación de remitir las cuestiones de interpretación, lo cual provocó una violación del apdo. 3 del art. 267. Locatelli (2018) ofrece una buena explicación de lo ocurrido en el sistema jurisdiccional administrativo francés. Una recopilación de estudios hechos en varios Estados miembros aparece en el muy buen artículo del juez Gervasoni del Tribunal General, publicado a modo de respuesta al artículo del presidente de la Sección de lo Contencioso del Conseild'État: Gervasoni (2019). 
jurisdiccional de la UE, insistiendo en la importancia de los derechos procesales nacionales para su funcionamiento y en el debido respeto a principios que garantizan la autoridad de las resoluciones judiciales. Esta jurisprudencia bien establecida le permite aquí responder bastante rápidamente y rechazar la necesidad de extender al ámbito del derecho de la UE este tipo de mecanismo de repetición del procedimiento penal.

Es interesante notar que, para apoyar su razonamiento, el Tribunal de Justicia emplea la efectividad de la protección de los derechos individuales en el sistema jurisdiccional de la Unión para establecer una distinción clara con el sistema de aplicación del CEDH. A pesar de que la efectividad del derecho de la Unión y de su asequibilidad para todos los justiciables desde el inicio de los procedimientos judiciales no es ideal, es cierto que es lo suficientemente buena como para evitar introducir mecanismos que ponen en tela de juicio la firmeza de las resoluciones nacionales. Desde una perspectiva más general, el Tribunal de Justicia se apoya en las características esenciales del sistema jurisdiccional de la UE y en un planteamiento constitucional, que sirve de base para el análisis de distintos mecanismos procesales, pero que sitúa estos mismos mecanismos en el centro del marco constitucional de la UE. En particular, el mecanismo de remisión prejudicial aparece, aquí como en la sentencia Associação Sindical dos Juizes Portugueses ${ }^{79}$ o en el Dictamen $2 / 13^{80}$, como un elemento esencial para el buen funcionamiento, no tan solo del sistema jurisdiccional, sino del ordenamiento jurídico de la UE. Esta sentencia pone de nuevo de manifiesto la afirmación, hecha por el Tribunal de Justicia, de su propio papel constitucional y de la importancia del sistema jurisdiccional y de la colaboración de los jueces nacionales para que pueda desempeñar este papel. Esta inclusión de los jueces de los Estados miembros en un sistema común, cuya razón de ser es la efectividad del proyecto de la UE, conlleva una supervisión de estos jueces por el Tribunal de Justicia. Este sigue buscando un equilibrio difícil entre cooperación en buena armonía con los jueces de los Estados miembros y un mayor énfasis en la jerarquía dentro del sistema jurisdiccional de la Unión. Sin embargo, como lo demuestra esta sentencia, esta inclusión también significa un reconocimiento de la importancia de la contribución de los sistemas judiciales nacional y aparece como un motivo de orgullo para el Tribunal de Justicia, ya que le permite insistir en la eficiencia de la protección de los derechos de los justiciables dentro de la Unión Europea.

79 Sentencia de 27 de febrero de 2018, apdo. 43.

80 Dictamen de 18 de diciembre de 2013, apdo. 176. 


\section{Bibliografía}

Adinolfi, A. (2012). The "Procedural Autonomy" of Member States and the Constraints Stemming from the ECJ's Case Law: Is Judicial Activism Still Necessary? En H. W. Micklitz y B. De Witte (eds.). The European Court of Justice and the Autonomy of the Member States (pp. 281-303). Cambridge: Intersentia.

Barav, A. (1993). Renvoi préjudiciel. En A. Barav, C. Philip y C. Boutayeb (eds.). Dictionnaire juridique des Communautés européennes (pp. 926-946). Paris: Presses Universitaires de France.

Bobek, M. (2012). Why there is no Principle of "Procedural Autonomy" of the Member States. En H. W. Micklitz y B. De Witte (eds.). The European Court of Justice and the Autonomy of the Member States (pp. 305-323). Cambridge: Intersentia.

Bourgeois, S. (2016). The Implementation of the European Convention on Human Rights at the Domestic Level. En S. Bourgeois et al. Comparative Study of the Implementation of the ECHR at the National Level (pp. 7-28). Belgrade: Council of Europe.

Bříza, P. (2008). Lucchini SpA-is There Anything Left of Res Judicata Principle? Civil Justice Quarterly, 27, 40-50.

Committee of Experts on the Reform of the Court (2016). Compilation of written contributions on the provision in the domestic legal order for re-examination or reopening of cases following judgments of the Court (DH-GDR(2015)002REV). Strasbourg: Council of Europe.

Gervasoni, S. (2019). CJUE et cours suprêmes: repenser les termes du dialogue des juges? Actualité Juridique Droit Administratif, 150.

Imbert, P. H. (2007). Convention européenne des droits de l'homme et ses protocoles. Jurisclasseur Libertés, 80.

Lagrange, M. (1974). L'action préjudicielle dans le droit interne des États membres et en droit communautaire. Revue Trimestrielle de Droit Européen, 10 (2), 268-297.

Lenaerts, K. et al. (2014). EU Procedural Law. Oxford: Oxford University Press.

Locatelli, F. (2018). Accor et désaccords - affaire dite du précompte mobilier: '[...] Et pour la première fois dans le cadre d'un recours en manquement [...]'. Droit fiscal, 41, 420 .

Martinico, G. (2012). Is the European Convention Going to Be Supreme? A Comparative-Constitutional Overview of ECHR and EU Law before National Courts. European Journal of International Law, 23 (2), 401-424. Disponible en: https:// doi.org/10.1093/ejil/chs027.

Peers, S. and Prechal, S. (2014). Article 52. En S. Peers, T. Hervey, J. Kenner y A. Ward (eds). The EU Charter of Fundamental Rights: A Commentary (pp. 14551522). Oxford: Hart.

Picod, F. (2002). La coopération juridictionnelle. En J. Auvret-Finck (dir.). L'Union européenne, carrefour des coopérations (pp. 199-232). Paris: LGDJ. 
Rubio Garrido, T. (2002). Cosa juzgada y tutela judicial efectiva. Derecho Privado y Constitución, 16, 259-391.

Sudre, F. (2016). Convention européenne des droits de l'Homme. Caractères généraux. Jurisclasseur Europe Traité, 6500.

Tizzano, A. y Gencarelli, B. (2011). Union Law and Final Decisions of National Courts in the Recent Case Law of the Court of Justice. En A. Arnull, C. Barnard, M. Dougan y E. Spaventa (eds). A Constitutional Order of States? Essays in EU Law in Honour of Alan Dashwood (pp. 267-280). Oxford: Hart.

Tomasin, D. (1975). Essai sur l'autorité de la chose jugée en matière civile. Paris: LGDJ.

Turmo, A. (2017). L'autorité de la chose jugée en droit de l'Union européenne. Bruxelles: Bruylant.

Wattel, P. J. (2004). Köbler, CILFIT and Welthgrove: We Can't Go on Meeting Like This. Common Market Law Review, 41, 177-190.

Weatherill, S. (2016). Law and Values in the European Union. Oxford: Oxford University Press. Disponible en: https://doi.org/10.1093/acprof:oso/9780199557264.001.0001. 